\title{
Note on the Lateral Displacement of Solid Particles and Gas in Shear Turbulent Flow
}

\author{
Gabrielie Asset and Dennis Phinlips
}

Chemical Research and Development Laboratories, Edgewood Arsenal, Maryland

5 February 1964 and 29 April 1964

\section{Introduction}

In studies of air pollution and the turbulent diffusion of small particles it is of interest to obtain data on the comparative turbulent diffusion of solids and gases. In this note are reported the results of measurements made of the lateral spread of solid particles and gas in a shear turbulent flow. Previous work has been carried out by Soo, Ihrig and ElKouh (1960) in studies of the turbulent diffusion of glass particles of 100 and 200 microns in diameter, and of helium gas in a fully developed channel flow. This note describes a study of turbulent diffusion of polystyrene particles ranging from 125 to 150 microns in diameter and having a specific gravity of 1.09 , and of ammonia gas in a flow consisting of a wake mixing with a turbulent boundary layer of a wind tunnel. The diffusion, studied close to the source, 10 to 63 inches, took place for short times ranging from 0.03 to 0.30 second. 


\section{Experimental procedures}

Fig. 1 is a schematic arrangement of the apparatus. In the wind tunnel, a copy of one described by Johnson (1957), the boundary layer was artificially thickened by means of 1/8-inch rods at the entry of the working section. The materials were injected into the boundary layer $12 \mathrm{ft}$ from the rods at a position where the turbulence was fully developed and the boundary layer thickness was 3.25 inches. The means of injection was a glass funnel which was clamped firmly to the top of the tunnel. A slit in the ceiling permitted the stem, 0.185 inch outside diameter and 0.035 inch inside diameter, to extend into the boundary layer at any desired position.

The polystyrene powder was slowly introduced into the boundary layer through the funnel stem at a flow rate of $1.2 \mathrm{cc}$ per $\mathrm{min}$. The mean free stream velocity was $21.3 \mathrm{mph}$, a speed too high for the particles to fall to the floor of the tunnel. The spheres were injected into the stream at various heights above the floor, 1.4, 2.4 and 6.4 inches, to test the effect of the intensity of turbulence. The position 6.4 inches above the floor was in the free-stream where the turbulence according to Johnson was very low. The particles were collected 13.1 inches and 63.3 inches downstream, always at the same height as the injector, in order to test the effect of time on diffusion. The means of collection was a single petroleum-coated steel wire, 0.015 inch in diameter, held taut horizontially at right angles to the direction of flow. The collecting efficiency of the wire was 100 per cent for particles of 100-150 microns moving at the velocity of $21.3 \mathrm{mph}$. After a collecting time of 1 minute the wire was removed from the tunnel and placed under a traveling microscope, where the number of spheres in an arbitrary area of $3.72 \times 10^{-4} \mathrm{sq}$. in. were counted at positions 0.1 inch apart along the wire.

The ammonia was injected into the flow approximately isokinetically through the funnel stem. The gas was injected 1.4 and 2.4 inches above the floor and collected 10.3 inches and 63.3 inches downstream from the disperser at somewhat higher levels, 1.7 inches and 1.9 inches, above the floor since it was found that the ammonia rose during its course dowastream. The collector consisted of a movable stainless steel probe of $1.1 \mathrm{~mm}$ inside diameter, which was connected to bubblers, containing a solution of $\mathrm{HCl}$. Samples of the diffused ammonia were drawn into the probe isokinetically by means of a pump for $1 \mathrm{~min}$ and at $1 / 8$-inch intervals across the tunnel at right angles to the stream. After the samples were collected the amount of ammonia in the bubblers was determined by a colorimetric method.

The wake of the stem was surveyed in the boundary layer 63.3 inches downstream from the funnel stem, while both the stem and the survey probe were $6.4,2.4$ and 1.4 inches above the floor. A survey was also made with ammonia issuing from the stem at heights of 1.9 and 1.7 inches. In the low turbulent region (6.4 inches above the floor) no significant velocity deficiency was

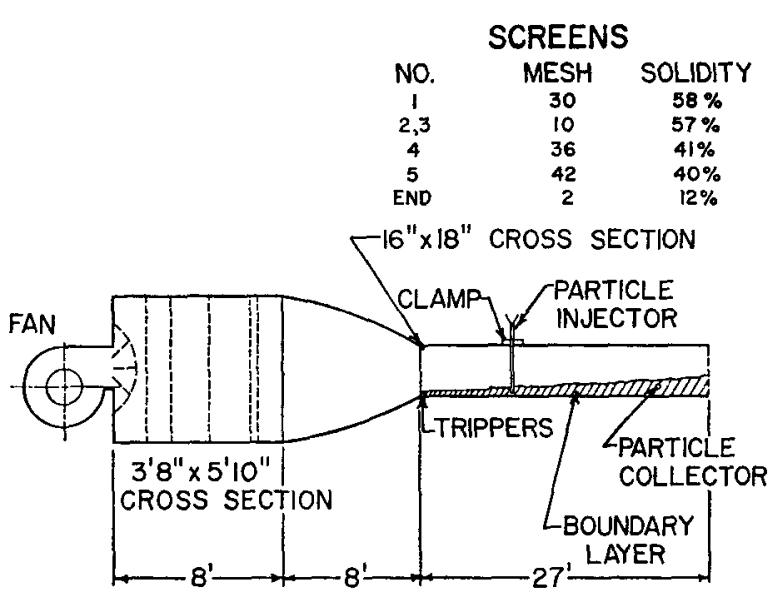

Fic. 1. Diagram of equipment.

present; but in the boundary layer a deficiency of about 3 per cent existed for the stem and of about 1 per cent for the stem with the jet of ammonia. The width $b_{0}$ of the wake was about 1.9 inches for both wakes.

\section{Treatment of data}

An examination of the concentration distributions of the particles along the wire collector and of ammonia across the tunnel lead to the assumption that both were Gaussian. Therefore the diffusion or spread of the material was treated as a variance in the mean displacement of the centroid of the material. The variance, or the mean square displacement, was computed by the usual group method.

The total spread, $\overline{Z^{2}}$, as determined experimentally, was due to several independent contributions. In the case of the polystyrene powder, $\overline{Z^{2}}$ was the sum of the contributions due to turbulence and to mutual electrostatic repulsion. In the case of ammonia, $\overline{Z^{2}}$ was the sum of the turbulent and the molecular diffusion. In either case

$$
\overline{Z^{2}}=\overline{Z_{t}}+\bar{Z}_{0}
$$

where $\bar{Z}_{t}$ was the mean square displacement due to turbulence, and $\bar{Z}_{o}$ is the displacement due to other causes. For the spheres $\overline{Z_{0}^{2}}$ was deternined experimentally in the section of the tunnel where the turbulence was low and for ammonia it was computed from the relation $\overline{Z_{o}^{2}}=2 \alpha t$ where $\alpha$ was the molecular diffusivity coefficient of ammonia, and $t$ was the time of diffusion. $\overline{Z_{t}^{2}}$ was computed for the spheres and the ammonia at the two downstream distances and the two distances above the floor. For ammonia, $t$ was computed from the relation $t=x U^{-1}$, where $x$ was the horizontal distance between the injector and the collector and $U$ was the mean wind velocity. Since the ammonia had been injected into the boundary isokinetically its velocity was $U$. The spheres however were introduced slowly into the 
flow in a vertical direction so that their initial horizontal velocity was zero. Since the particles because of their inertia traveled more slowly than the air, the time of diffusion was calculated as $x(U-q)^{-1}$, where $q$ was the difference between the mean wind velocity and the particle velocity. Values of $q$ for different particle sizes and wind velocities were obtained from the report of Soo et al. (1960).

\section{Discussion of results}

The mean square displacement, $\overline{Z_{t}^{2}}$, due to turbulence, depended on the distance above the floor and the time of diffusion (Table 1 ). The results indicated that $\overline{Z_{t}^{2}}$ was a function of a time parameter $t d y^{-1}$ where $d$ is the boundary thickness determined from the velocity profiles. $\overline{Z_{t}^{2}}$ was an increasing monotonic function of $t d y^{-1}$ (see Fig. 2), which increased more rapidly for ammonia than for the spheres. The ratio of $\overline{Z_{t}^{2}}$ for ammonia to $\overline{Z_{t}^{2}}$ for spheres for $t d y^{-1}=0.1$ and 0.4 second were 4.3 and 4.1 , respectively. In view of the fact that the standard deviation of $\overline{Z_{t}{ }^{2}}$ was 10 per cent of the mean these ratios are not significantly different.

Soo obtained a value of 0.17 in. $^{2}$ for $\overline{Z_{t}^{2}}$, by using helium as the diffusing gas, we obtained 0.16 in. ${ }^{2}$ with ammonia for the same time of diffusion $(0.03 \mathrm{sec})$. Despite the difference in methods, in flows, and the spread of data in the experiments, these values are of the same order of magnitude.

Soo found the turbulent diffusity, $D$, of 200 -micron glass beads in a flow of $30 \mathrm{ft} \mathrm{sec}-1$ to be $0.158 \mathrm{in}^{2} \mathrm{sec}^{-1}$

TaBLE 1. Lateral turbulent displacement of particles and gas.

\begin{tabular}{cccccc}
\hline & & & & & \\
Collector & Position & $t$ & $\overline{Z_{0}^{2}}$ & $\overline{Z_{t}^{2}}$ & $\theta$ \\
\hline$x$ in. & $y$ in. & sec & in. $^{2}$ & in. $^{2}$ & in. $^{2}$
\end{tabular}

A. Polystyrene spheres, $125-150 \mu$ diameter

\begin{tabular}{llllll}
13.1 & 6.4 & & 0.050 & & \\
& 2.4 & 0.048 & 0.050 & 0.026 & 0.019 \\
& 1.4 & 0.058 & 0.050 & 0.101 & 0.022 \\
63.3 & 6.4 & & 0.050 & & \\
& 2.4 & 0.24 & 0.050 & 0.426 & 0.092 \\
& 1.4 & 0.29 & 0.050 & 0.898 & 0.110 \\
& & \multicolumn{5}{c}{ B. Ammonia (gas) } \\
& & & \\
& & & \\
10.3 & 2.6 & 0.027 & 0.005 & 0.104 & 0.011 \\
& 1.7 & 0.031 & 0.006 & 0.158 & 0.012 \\
63.3 & 1.9 & 0.19 & 0.035 & 1.88 & 0.19
\end{tabular}

$x=$ distance downwind from the ejector.

$y=$ distance above the floor of the tunnel.

$t=$ time of diffusion.

$\overline{Z_{0}^{2}}=$ mean square displacement due to factors other than turbulence.

$\overline{Z^{2}}=$ mean square displacement due to turbulence. $\theta=$ standard deviation of $\overline{Z_{t}}$.

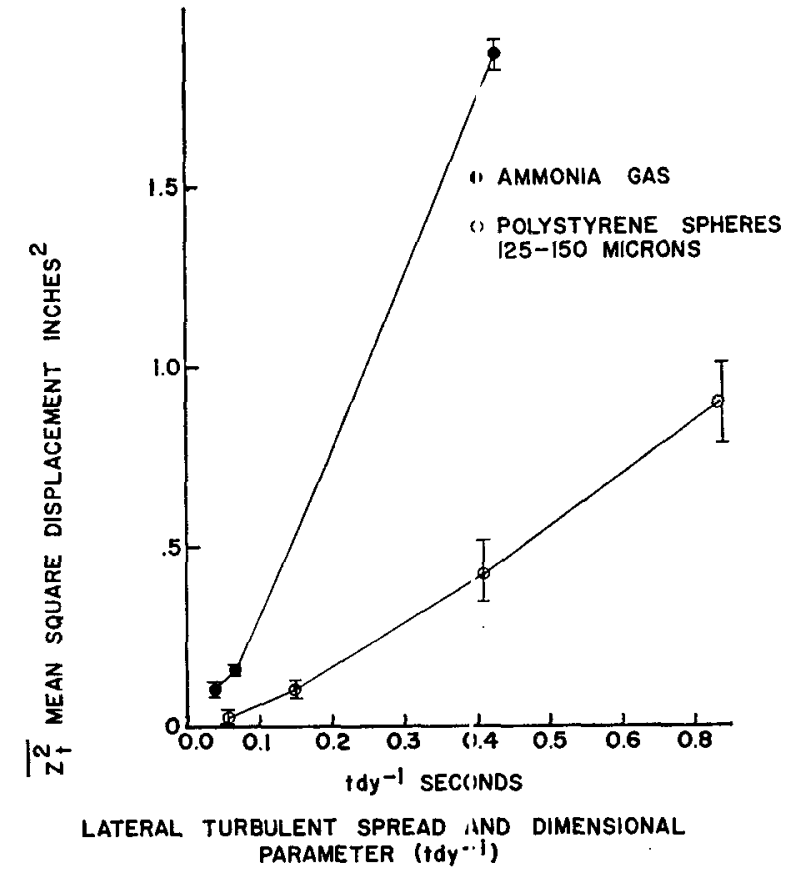

Fu. 2. Lateral turbulent spread and dimensional parameter $\left(t d y^{-1}\right)$ versus mean square displacemınt (inch $\left.{ }^{2}\right)$ for ammonia gas and polystyrene spheres.

For polystyrene beads of 125 nicron in a flow of the same velocity $D$ was 3.4 in. $^{2}$ ser $^{-1}$, when computed by the relationship $D=\frac{1}{2} d \overline{Z^{2}} / d t$. The difference is one of an order of magnitude which cannot be explained by the difference of particle densities which is of the same order. A better explanation of this difference can be found in a consideration of the tr.rbulent structure of the two types of flow. In the channel flow of Soo the turbulent intensity was 2-3 per cent, which was actually measured and found to be uniform throughout the working section of the channel. The integral scale of turbulence may be estimated to be $\frac{1}{2}$ the channel width or 1.5 inches. On the other hand in the wake mixing in a turbulent boundary layer the scale and intensity were estimated from results by Eskinazi (1959). The turbulent intensity within the wake was 20 per cent at the center and 9 per cent at the edges at a distance downstream of 1.0 inch from the funn 31 stem; while it decayed to 7-8 per cent farther downstream. The integral scale of the wake was 0.16 to 1.2 inches at a distance 1 inch downstream from the ejector, and 1.6 inches farther downstream. It would seem threfore that the gas diffusion was affected by the presence of turbulence of smaller intensity (3-5 per cer $t$ ) and greater integral scale (1.5 and 1.6 inch) while the particle diffusion was affected to a greater extent by the presence of turbulence of greater intensity (9-2J per cent) and smaller scale $(0.16$ inch). 


\section{REFERENCES}

Eskanazi, S., 1959: Mixing of wakes in a turbulent shear flow. NASA Tech. Note D-83, $33 \mathrm{pp}$.

Johnson, D. S., 1957: Velocity, temperature and heat-transfer measurements in a turbulent boundary layer downstream of a stepwise discontinuity in wall temperature. $J . a p p l$. Mech., $24,1-7$.

Soo, S. L., H. K. Ihrig and A. F. ElKouh, 1960: Experimental determination of statistical properties of two-phase turbulent motion. ASME Trans. J. Basic Eng., 82, ser. D, 609-621. 